\title{
ON SOME HERMITE-HADAMARD TYPE INEQUALITIES FOR TWICE DIFFERENTIABLE MAPPINGS AND APPLICATIONS
}

\author{
U. S. KIRMACI AND R. DİKİCİ
}

\begin{abstract}
Some inequalities for twice differentiable mappings are presented. Some applications to special means of real numbers are also given.
\end{abstract}

\section{Introduction}

A function $f: I \subset R \rightarrow R$ is called convex on the interval $I$ of real numbers if

$$
f(\lambda x+(1-\lambda) y) \leq \lambda f(x)+(1-\lambda) f(y)
$$

for all points $x$ and $y$ in $I$ and all $\lambda \in[0,1]$.

Let $f: I \subset R \rightarrow R$ be a convex function on the interval $I$ of real numbers and $a, b \in I$ with $a<b$. The inequality

$$
f\left(\frac{a+b}{2}\right) \leq \frac{1}{b-a} \int_{a}^{b} f(x) d x \leq \frac{f(a)+f(b)}{2}
$$

is known as Hermite-Hadamard's inequality for convex functions [7].

In 1935, G. Grüss (see [7, p.70]) proved the following integral inequality which gives an approximation for the integral of a product of two functions in terms of the product of integrals of the two functions.

Theorem A. Let $h, g:[a, b] \rightarrow R$ be two integrable functions such that $\phi_{1} \leq h(x) \leq \Phi_{1}$ and $\gamma_{1} \leq g(x) \leq \Gamma_{1}$ for all $x \in[a, b]$, where $\phi_{1}, \Phi_{1}, \gamma_{1}, \Gamma_{1}$, are real numbers. Then we have

$$
|T(h, g)|:=\left|\frac{1}{b-a} \int_{a}^{b} h(x) g(x) d x-\frac{1}{b-a} \int_{a}^{b} h(x) d x \cdot \frac{1}{b-a} \int_{a}^{b} g(x) d x\right| \leq \frac{1}{4}\left(\Phi_{1}-\phi_{1}\right)\left(\Gamma_{1}-\gamma_{1}\right)
$$

and the inequality is sharp, in the sense that the constant $1 / 4$ cannot be replaced by a smaller one.

In [7, p.40], Chebychev's inequlity is given by the following theorem:

Corresponding author: U. S. Kirmaci.

Key words and phrases. Convex functions, Hölder's inequality, Grüss' inequality, Chebychev's inequality, Bullen's inequality, Special means. 
Theorem B. Let $f$ and $g$ be two functions which are integrable and monotone in the same sense on $(a, b)$ and let $p$ be a positive and integrable function on the same interval. Then,

$$
\int_{a}^{b} p(x) f(x) g(x) d x \int_{a}^{b} p(x) d x \geq \int_{a}^{b} p(x) f(x) d x \cdot \int_{a}^{b} p(x) g(x) d x
$$

with equality if and only if one of the functions $f, g$ reduces to a constant.

If $f$ and $g$ are monotone in the opposite sense, the reverse inequality holds.

Let $f: I \subset R \rightarrow R$ be a convex function on the interval $I$ of real numbers and $a, b \in I$ with $a<b$. The inequality

$$
\frac{1}{b-a} \int_{a}^{b} f(x) d x \leq \frac{1}{2}\left[f\left(\frac{a+b}{2}\right)+\frac{f(a)+f(b)}{2}\right]
$$

is known as Bullen's inequality for convex functions [1, p.39].

In the literature, the following definition is well known:

Let $f:[a, b] \rightarrow R$ and $p \in R^{+}$. The $p$-norm of the function $f$ on $[a, b]$ is defined by

$$
\|f\|_{p} \begin{cases}\left(\int_{a}^{b}|f(x)|^{p} d x\right)^{1 / p}, & 0<p<\infty \\ \sup |f(x)|, & p=\infty,\end{cases}
$$

and $L^{p}([a, b])$ is the set of all functions $f:[a, b] \rightarrow R$ such that $\|f\|_{p}<\infty$.

For several recent results concerning Hermite-Hadamard's inequality and twice differentiable mappings, we refer the reader to [1]-[6].

In this paper, we give new inequalities for twice differentiable mappings and some applications to special means of real numbers.

\section{Main results}

We first prove the following lemma:

Lemma 1. Let $f: I \subset R \rightarrow R$ be twice differentiable mapping on $I^{0}$ such that $f^{\prime \prime} \in L^{\prime}[a, b]$, where $a, b \in I^{0}$ with $a<b$. Then we have the equality

$$
\begin{aligned}
& \frac{1}{2(b-a)}\left[\int_{a}^{\frac{a+b}{2}}(x-a)\left(\frac{a+b}{2}-x\right) f^{\prime \prime}(x) d x+\int_{\frac{a+b}{2}}^{b}(b-x)\left(x-\frac{a+b}{2}\right) f^{\prime \prime}(x) d x\right] \\
& =\frac{1}{2}\left[f\left(\frac{a+b}{2}\right)+\frac{f(a)+f(b)}{2}\right]-\frac{1}{b-a} \int_{a}^{b} f(x) d x
\end{aligned}
$$

where, $I^{0}$ denotes the interior of I. 
Proof. By integration by parts twice, we have

$$
\begin{aligned}
\int_{a}^{\frac{a+b}{2}} & (x-a)\left(\frac{a+b}{2}-x\right) f^{\prime \prime}(x) d x+\int_{\frac{a+b}{2}}^{b}(b-x)\left(x-\frac{a+b}{2}\right) f^{\prime \prime}(x) d x \\
& =\int_{a}^{\frac{a+b}{2}}\left[2 x-\left(\frac{a+b}{2}+a\right)\right] f^{\prime}(x) d x+\int_{\frac{a+b}{2}}^{b}\left[2 x-\left(\frac{a+b}{2}+b\right)\right] f^{\prime}(x) d x \\
& =(b-a)\left[f\left(\frac{a+b}{2}\right)+\frac{f(a)+f(b)}{2}\right]-2 \int_{a}^{b} f(x) d x
\end{aligned}
$$

Hence, we obtain desired equality (1).

Theorem 1. Let $f: I \subset R \rightarrow R$ be twice differentiable mapping on $I^{0}$ such that $f^{\prime \prime} \in L^{\prime}[a, b]$, where $a, b \in I^{0}$ with $a<b$. If the mapping

$$
\varphi(x)= \begin{cases}(x-a)\left(\frac{a+b}{2}-x\right) f^{\prime \prime}(x), & x \in\left[a, \frac{a+b}{2}\right), \\ (b-x)\left(x-\frac{a+b}{2}\right) f^{\prime \prime}(x), & x \in\left[\frac{a+b}{2}, b\right],\end{cases}
$$

is convex on $[a, b]$, then we have the inequality

$$
\begin{aligned}
\frac{(b-a)^{2}}{64}\left[f^{\prime \prime}\left(\frac{3 a+b}{4}\right)+f^{\prime \prime}\left(\frac{a+3 b}{4}\right)\right] & \leq \frac{1}{2}\left[f\left(\frac{a+b}{2}\right)+\frac{f(a)+f(b)}{2}\right]-\frac{1}{b-a} \int_{a}^{b} f(x) d x \\
& \leq \frac{(b-a)^{2}}{128}\left[f^{\prime \prime}\left(\frac{3 a+b}{4}\right)+f^{\prime \prime}\left(\frac{a+3 b}{4}\right)\right] .
\end{aligned}
$$

Proof. Applying the first inequality of Hermite-Hadamard for the mapping $\varphi$, we write

and

$$
\frac{2}{b-a} \int_{a}^{\frac{a+b}{2}} \varphi(x) d x \geq \varphi\left(\frac{3 a+b}{4}\right)=\frac{(b-a)^{2}}{16} f^{\prime \prime}\left(\frac{3 a+b}{4}\right),
$$

$$
\frac{2}{b-a} \int_{\frac{a+b}{2}}^{b} \varphi(x) d x \geq \varphi\left(\frac{a+3 b}{4}\right)=\frac{(b-a)^{2}}{16} f^{\prime \prime}\left(\frac{a+3 b}{4}\right) .
$$

Applying the Bullen's inequality for the mapping $\varphi$, we have

$$
\frac{2}{b-a} \int_{a}^{\frac{a+b}{2}} \varphi(x) d x \leq \frac{1}{2}\left[\varphi\left(\frac{3 a+b}{4}\right)+\frac{\varphi(a)+\varphi\left(\frac{a+b}{2}\right)}{2}\right]=\frac{(b-a)^{2}}{32} f^{\prime \prime}\left(\frac{3 a+b}{4}\right),
$$

and

$$
\frac{2}{b-a} \int_{\frac{a+b}{2}}^{b} \varphi(x) d x \leq \frac{1}{2}\left[\varphi\left(\frac{a+3 b}{4}\right)+\frac{\varphi\left(\frac{a+b}{2}\right)+\varphi(b)}{2}\right]=\frac{(b-a)^{2}}{32} f^{\prime \prime}\left(\frac{a+3 b}{4}\right) .
$$

Adding all these inequalities and from Lemma 1, we have

$$
\frac{(b-a)^{2}}{64}\left[f^{\prime \prime}\left(\frac{3 a+b}{4}\right)+f^{\prime \prime}\left(\frac{a+3 b}{4}\right)\right] \leq \frac{1}{2}\left[f\left(\frac{a+b}{2}\right)+\frac{f(a)+f(b)}{2}\right]-\frac{1}{b-a} \int_{a}^{b} f(x) d x
$$




$$
\leq \frac{(b-a)^{2}}{128}\left[f^{\prime \prime}\left(\frac{3 a+b}{4}\right)+f^{\prime \prime}\left(\frac{a+3 b}{4}\right)\right]
$$

which is required inequality (2).

Example. Let us consider function $f$ defined as $f(x)=x^{3}$. Then we have $f^{\prime \prime}(x)=6 x$. If we apply Theorem 1, we obtain

$$
\frac{3(b-a)^{2}(a+b)}{32} \leq \frac{1}{2}\left[f\left(\frac{a+b}{2}\right)+\frac{f(a)+f(b)}{2}\right]-\frac{1}{b-a} \int_{a}^{b} f(x) d x \leq \frac{3(b-a)^{2}(a+b)}{64} .
$$

If we choose $a=-2, b=1$, we deduce

$$
-\frac{27}{32} \leq \frac{1}{2}\left[f\left(\frac{a+b}{2}\right)+\frac{f(a)+f(b)}{2}\right]-\frac{1}{b-a} \int_{a}^{b} f(x) d x \leq-\frac{27}{64}
$$

where

$$
\frac{1}{2}\left[f\left(\frac{a+b}{2}\right)+\frac{f(a)+f(b)}{2}\right]-\frac{1}{b-a} \int_{a}^{b} f(x) d x=-\frac{9}{16} .
$$

Theorem 2. Let $f: I \subset R \rightarrow R$ be twice differentiable mapping on $I^{0}, p>1$, and $q=\frac{p}{p-1}$. If $\left|f^{\prime \prime}\right| \in L^{\prime}[a, b]$, where $a, b \in I^{0}$ with $a<b$, then we have the inequality

$$
\left|\frac{1}{2}\left[f\left(\frac{a+b}{2}\right)+\frac{f(a)+f(b)}{2}\right]-\frac{1}{b-a} \int_{a}^{b} f(x) d x\right| \leq \frac{(b-a)^{(p+1) / p}}{2^{(2 p+1) / p}}[B(p+1, p+1)]^{1 / p}\left\|f^{\prime \prime}\right\|_{q}
$$

where, $B(p, q)$ is Euler's Beta function.

Proof. By (1) and Hölder's inequality, we have that

$$
\begin{aligned}
\mid \frac{1}{2}[ & \left.f\left(\frac{a+b}{2}\right)+\frac{f(a)+f(b)}{2}\right]-\frac{1}{b-a} \int_{a}^{b} f(x) d x \mid \\
\leq & \left|\frac{1}{2(b-a)}\left[\int_{a}^{\frac{a+b}{2}}(x-a)\left(\frac{a+b}{2}-x\right) f^{\prime \prime}(x) d x+\int_{\frac{a+b}{2}}^{b}(b-x)\left(x-\frac{a+b}{2}\right) f^{\prime \prime}(x) d x\right]\right| \\
\leq & \frac{1}{2(b-a)}\left[\left(\int_{a}^{\frac{a+b}{2}}(x-a)^{p}\left(\frac{a+b}{2}-x\right)^{p}\right)^{1 / p}\left(\int_{a}^{\frac{a+b}{2}}\left|f^{\prime \prime}(x)\right|^{q} d x\right)^{1 / q}\right. \\
& \left.+\left(\int_{\frac{a+b}{2}}^{b}(b-x)^{p}\left(x-\frac{a+b}{2}\right)^{p}\right)^{1 / p}\left(\int_{\frac{a+b}{2}}^{b}\left|f^{\prime \prime}(x)\right|^{q} d x\right)^{1 / q}\right] .
\end{aligned}
$$

Using the change of the variable $x=(1-t) a+t\left(\frac{a+b}{2}\right)$ and from $d x=\left(\frac{b-a}{2}\right) d t$, we write

$$
\begin{aligned}
\int_{a}^{\frac{a+b}{2}} & (x-a)^{p}\left(\frac{a+b}{2}-x\right)^{p} d x \\
\quad & =\left(\frac{b-a}{2}\right) \int_{0}^{1}\left((1-t) a+t\left(\frac{a+b}{2}\right)-a\right)^{p}\left(\frac{a+b}{2}-(1-t) a-t\left(\frac{a+b}{2}\right)\right)^{p} d t \\
& =\left(\frac{b-a}{2}\right)^{2 p+1} \int_{0}^{1} t^{p}(1-t)^{p} d t=\left(\frac{b-a}{2}\right)^{2 p+1} B(p+1, p+1)
\end{aligned}
$$


where, $B(p, q)=\int_{0}^{1} t^{p-1}(1-t)^{q-1} d t,(p, q>0)$.

Using the change of the variable $x=(1-t) \frac{a+b}{2}+t b$ and from $d x=\left(\frac{b-a}{2}\right) d t$, we write

$$
\begin{aligned}
\int_{\frac{a+b}{2}}^{b}(b-x)^{p}\left(x-\frac{a+b}{2}\right)^{p} d x & =\left(\frac{b-a}{2}\right) \int_{0}^{1}\left(b-(1-t) \frac{a+b}{2}-t b\right)^{p}\left((1-t) \frac{a+b}{2}+t b-\frac{a+b}{2}\right)^{p} d t \\
& =\left(\frac{b-a}{2}\right)^{2 p+1} \int_{0}^{1} t^{p}(1-t)^{p} d t=\left(\frac{b-a}{2}\right)^{2 p+1} B(p+1, p+1) .
\end{aligned}
$$

Combining all this inequalities, we have

$$
\left|\frac{1}{2}\left[f\left(\frac{a+b}{2}\right)+\frac{f(a)+f(b)}{2}\right]-\frac{1}{b-a} \int_{a}^{b} f(x) d x\right| \leq \frac{(b-a)^{(p+1) / p}}{2^{(2 p+1) / p}}\left[B(p+1, p+1]^{1 / p}\left\|f^{\prime \prime}\right\|_{q} .\right.
$$

Hence, the theorem is proved.

Remark. Let $f$ be as in Theorem 2. For $p>1, p \in N$ and using the equality

$$
B(p+1, p+1)=\frac{p !}{(p+1) \cdots(2 p+1)}=\frac{[p !]^{2}}{(2 p+1) !}
$$

we deduce

$$
\left|\frac{1}{2}\left[f\left(\frac{a+b}{2}\right)+\frac{f(a)+f(b)}{2}\right]-\frac{1}{b-a} \int_{a}^{b} f(x) d x\right| \leq \frac{(b-a)^{(p+1) / p}}{2^{(2 p+1) / p}}\left[\frac{[p !]^{2}}{(2 p+1) !}\right]^{1 / p}\left\|f^{\prime \prime}\right\|_{q}
$$

which gives for $p=2$ that

$$
\left|\frac{1}{2}\left[f\left(\frac{a+b}{2}\right)+\frac{f(a)+f(b)}{2}\right]-\frac{1}{b-a} \int_{a}^{b} f(x) d x\right| \leq \frac{\sqrt{960}}{960}(b-a)^{3 / 2}\left\|f^{\prime \prime}\right\|_{2} .
$$

Theorem 3. Let $f: I \subset R \rightarrow R$ be twice differentiable mapping on $I^{0}$ such that $\gamma \leq f^{\prime \prime}(x) \leq \mu$ on $\left[a, \frac{a+b}{2}\right) \subset I^{0}$ and $\gamma^{\prime} \leq f^{\prime \prime}(x) \leq \mu^{\prime}$ on $\left[\frac{a+b}{2}, b\right] \subset I^{0}$. If $f^{\prime \prime} \in L^{\prime}[a, b]$, then we have the inequality $\left|\frac{1}{2}\left[f\left(\frac{a+b}{2}\right)+\frac{f(a)+f(b)}{2}\right]-\frac{1}{b-a} \int_{a}^{b} f(x) d x-\frac{b-a}{96}\left[f^{\prime}(b)-f^{\prime}(a)\right]\right| \leq \frac{(b-a)^{2}}{128}\left[(\mu-\gamma)+\left(\mu^{\prime}-\gamma^{\prime}\right)\right]$

Proof. By Grüss inequality, we have that

$$
\begin{aligned}
& \left|\Delta_{1}-\left(\frac{1}{b-a}\right)^{2}\left[\int_{a}^{\frac{a+b}{2}}(x-a)\left(\frac{a+b}{2}-x\right) d x \int_{a}^{\frac{a+b}{2}} f^{\prime \prime}(x) d x+\int_{\frac{a+b}{2}}^{b}(b-x)\left(x-\frac{a+b}{2}\right) d x \int_{\frac{a+b}{2}}^{b} f^{\prime \prime}(x) d x\right]\right| \\
& \quad \leq \frac{1}{4}\left[(\ell-l)(\mu-\gamma)+\left(\ell^{\prime}-l^{\prime}\right)\left(\mu^{\prime}-\gamma^{\prime}\right)\right]
\end{aligned}
$$

where

$$
\ell=\sup _{x \in\left[a, \frac{a+b}{2}\right]}\left\{(x-a)\left(\frac{a+b}{2}-x\right)\right\}=\frac{(b-a)^{2}}{16}, \quad l=\inf _{x \in\left[a, \frac{a+b}{2}\right]}\left\{(x-a)\left(\frac{a+b}{2}-x\right)\right\}=0,
$$




$$
\ell^{\prime}=\sup _{x \in\left[\frac{a+b}{2}, b\right]}\left\{(b-x)\left(x-\frac{a+b}{2}\right)\right\}=\frac{(b-a)^{2}}{16}, \quad l^{\prime}=\inf _{x \in\left[\frac{a+b}{2}, b\right]}\left\{(b-x)\left(x-\frac{a+b}{2}\right)\right\}=0 .
$$

By integration by parts, we get

$$
\int_{a}^{\frac{a+b}{2}}(x-a)\left(\frac{a+b}{2}-x\right) d x=\int_{\frac{a+b}{2}}^{b}(b-x)\left(x-\frac{a+b}{2}\right) d x=\frac{(b-a)^{3}}{48} .
$$

By Lemma 1, we have that

$$
\Delta=\frac{1}{2}\left[f\left(\frac{a+b}{2}\right)+\frac{f(a)+f(b)}{2}\right]-\frac{1}{b-a} \int_{a}^{b} f(x) d x
$$

where $\Delta=\frac{1}{2} \Delta_{1}$. Hence, we obtain,

$$
\begin{aligned}
\mid \Delta & -\frac{1}{2}\left(\frac{1}{(b-a)^{2}}\right) \frac{(b-a)^{3}}{48}\left\{\left[f^{\prime}\left(\frac{a+b}{2}\right)-f^{\prime}(a)\right]+\left[f^{\prime}(b)-f^{\prime}\left(\frac{a+b}{2}\right)\right]\right\} \mid \\
& \leq \frac{(b-a)^{2}}{128}\left[(\mu-\gamma)+\left(\mu^{\prime}-\gamma^{\prime}\right)\right] .
\end{aligned}
$$

That is,

$$
\left|\Delta-\frac{b-a}{96}\left[f^{\prime}(b)-f^{\prime}(a)\right]\right| \leq \frac{(b-a)^{2}}{128}\left[(\mu-\gamma)+\left(\mu^{\prime}-\gamma^{\prime}\right)\right]
$$

and the theorem is proved.

Theorem 4. Let $f: I \subset R \rightarrow R$ be twice differentiable mapping on $I^{0}$. If $f^{\prime \prime} \in L^{\prime}[a, b]$ and $k \leq$ $f^{\prime \prime}(x) \leq K$ for all $x \in[a, b] \subset I^{0}$, then we have the inequality

$$
K \frac{(b-a)^{2}}{48}-\frac{1}{2(b-a)}\left(\frac{\sigma_{1} \sigma_{2}}{\sigma_{3}}+\frac{\sigma_{4} \sigma_{5}}{\sigma_{6}}\right) \leq I_{1}+I_{2} \leq k \frac{(b-a)^{2}}{48}-\frac{1}{2(b-a)}\left(\frac{\alpha_{1} \alpha_{2}}{\alpha_{3}}+\frac{\alpha_{4} \alpha_{5}}{\alpha_{6}}\right)
$$

where,

$$
I_{1}+I_{2}=\frac{1}{2}\left[f\left(\frac{a+b}{2}\right)+\frac{f(a)+f(b)}{2}\right]-\frac{1}{b-a} \int_{a}^{b} f(x) d x
$$

and $\alpha_{i}, \sigma_{i},(i=1, \ldots, 6)$ are given by (12)-(17) and (18)-(23) respectively.

Proof. By Lemma 1, we have that

$$
\Delta=I_{1}+I_{2}=\frac{1}{2}\left[f\left(\frac{a+b}{2}\right)+\frac{f(a)+f(b)}{2}\right]-\frac{1}{b-a} \int_{a}^{b} f(x) d x
$$

where

$$
\begin{aligned}
& I_{1}=\frac{1}{2(b-a)} \int_{a}^{\frac{a+b}{2}}(x-a)\left(\frac{a+b}{2}-x\right) f^{\prime \prime}(x) d x, \\
& I_{2}=\frac{1}{2(b-a)} \int_{\frac{a+b}{2}}^{b}(b-x)\left(x-\frac{a+b}{2}\right) f^{\prime \prime}(x) d x .
\end{aligned}
$$


By integration by parts, we deduce

$$
\begin{aligned}
\frac{1}{2(b-a)} \int_{a}^{\frac{a+b}{2}}(x-a)\left(\frac{a+b}{2}-x\right)\left(f^{\prime \prime}(x)-k\right) d x & =I_{1}-k \frac{1}{2(b-a)} \int_{a}^{\frac{a+b}{2}}(x-a)\left(\frac{a+b}{2}-x\right) d x \\
& =I_{1}-k \frac{(b-a)^{2}}{96}
\end{aligned}
$$

and

$$
\frac{1}{2(b-a)} \int_{a}^{\frac{a+b}{2}}(x-a)\left(\frac{a+b}{2}-x\right)\left(K-f^{\prime \prime}(x)\right) d x=K \frac{(b-a)^{2}}{96}-I_{1} .
$$

Also, we have

$$
\begin{aligned}
& \frac{1}{2(b-a)} \int_{\frac{a+b}{2}}^{b}(b-x)\left(x-\frac{a+b}{2}\right)\left(f^{\prime \prime}(x)-k\right) d x=I_{2}-k \frac{(b-a)^{2}}{96}, \\
& \frac{1}{2(b-a)} \int_{\frac{a+b}{2}}^{b}(b-x)\left(x-\frac{a+b}{2}\right)\left(K-f^{\prime \prime}(x)\right) d x=K \frac{(b-a)^{2}}{96}-I_{2} .
\end{aligned}
$$

By Chebychev integral inequality, we have the following inequalities:

$$
\begin{aligned}
& \frac{1}{2(b-a)} \int_{a}^{\frac{a+b}{2}}(x-a)\left(\frac{a+b}{2}-x\right)\left(f^{\prime \prime}(x)-k\right) d x \\
& \leq \frac{1}{2(b-a)} \frac{\int_{a}^{\frac{a+b}{2}}(x-a)\left(f^{\prime \prime}(x)-k\right) d x \int_{a}^{\frac{a+b}{2}}\left(\frac{a+b}{2}-x\right)\left(f^{\prime \prime}(x)-k\right) d x}{\int_{a}^{\frac{a+b}{2}}\left(f^{\prime \prime}(x)-k\right) d x} \\
& \frac{1}{2(b-a)} \int_{\frac{a+b}{2}}^{b}(b-x)\left(x-\frac{a+b}{2}\right)\left(f^{\prime \prime}(x)-k\right) d x \\
& \leq \frac{1}{2(b-a)} \frac{\int_{\frac{a+b}{2}}^{b}(b-x)\left(f^{\prime \prime}(x)-k\right) d x \int_{\frac{a+b}{2}}^{b}\left(x-\frac{a+b}{2}\right)\left(f^{\prime \prime}(x)-k\right) d x}{\int_{\frac{a+b}{2}}^{b}\left(f^{\prime \prime}(x)-k\right) d x} \\
& \frac{1}{2(b-a)} \int_{a}^{\frac{a+b}{2}}(x-a)\left(\frac{a+b}{2}-x\right)\left(K-f^{\prime \prime}(x)\right) d x \\
& \leq \frac{1}{2(b-a)} \frac{\int_{a}^{\frac{a+b}{2}}(x-a)\left(K-f^{\prime \prime}(x)\right) d x \int_{a}^{\frac{a+b}{2}}\left(\frac{a+b}{2}-x\right)\left(K-f^{\prime \prime}(x)\right) d x}{\int_{a}^{\frac{a+b}{2}}\left(K-f^{\prime \prime}(x)\right) d x} \\
& \frac{1}{2(b-a)} \int_{\frac{a+b}{2}}^{b}(b-x)\left(x-\frac{a+b}{2}\right)\left(K-f^{\prime \prime}(x)\right) d x \\
& \leq \frac{1}{2(b-a)} \frac{\int_{\frac{a+b}{2}}^{b}(b-x)\left(K-f^{\prime \prime}(x)\right) d x \int_{\frac{a+b}{2}}^{b}\left(x-\frac{a+b}{2}\right)\left(K-f^{\prime \prime}(x)\right) d x}{\int_{\frac{a+b}{2}}^{b}\left(K-f^{\prime \prime}(x)\right) d x} .
\end{aligned}
$$

By integration by parts, we have the following equalities:

$$
\int_{a}^{\frac{a+b}{2}}(x-a)\left(f^{\prime \prime}(x)-k\right) d x
$$




$$
\begin{aligned}
& =\left[f^{\prime}\left(\frac{a+b}{2}\right)\right]-k\left(\frac{a+b}{2}\right)\left(\frac{b-a}{2}\right)-\left(f\left(\frac{a+b}{2}\right)-f(a)+k\left(\frac{(a+b)^{2}}{8}-\frac{a^{2}}{2}\right)\right)=\alpha_{1} \\
& \int_{a}^{\frac{a+b}{2}}\left(\frac{a+b}{2}-x\right)\left(f^{\prime \prime}(x)-k\right) d x \\
& =\left(k a-f^{\prime}(a)\right)\left(\frac{b-a}{2}\right)+\left[f\left(\frac{a+b}{2}\right)-f(a)-k\left(\frac{(a+b)^{2}}{8}-\frac{a^{2}}{2}\right)\right]=\alpha_{2} \\
& \int_{a}^{\frac{a+b}{2}}\left(f^{\prime \prime}(x)-k\right) d x=f^{\prime}\left(\frac{a+b}{2}\right)-f^{\prime}(a)-k\left(\frac{b-a}{2}\right)=\alpha_{3} \\
& \int_{\frac{a+b}{2}}^{b}(b-x)\left(f^{\prime \prime}(x)-k\right) d x \\
& =\left[k\left(\frac{a+b}{2}\right)-f^{\prime}\left(\frac{a+b}{2}\right)\right]\left(\frac{b-a}{2}\right)+f(b)-f\left(\frac{a+b}{2}\right)-k\left(\frac{b^{2}}{2}-\frac{(a+b)^{2}}{8}\right)=\alpha_{4} \\
& \int_{\frac{a+b}{2}}^{b}\left(x-\frac{a+b}{2}\right)\left(f^{\prime \prime}(x)-k\right) d x \\
& =\left(f^{\prime}(b)-k b\right)\left(\frac{b-a}{2}\right)-\left[f(b)-f\left(\frac{a+b}{2}\right)-k\left(\frac{(a+b)^{2}}{8}-\frac{b^{2}}{2}\right)\right]=\alpha_{5} \\
& \int_{\frac{a+b}{2}}^{b}\left(f^{\prime \prime}(x)-k\right) d x=f^{\prime}(b)-f^{\prime}\left(\frac{a+b}{2}\right)-k\left(\frac{b-a}{2}\right)=\alpha_{6} \\
& \int_{a}^{\frac{a+b}{2}}(x-a)\left(K-f^{\prime \prime}(x)\right) d x \\
& =-\left[f^{\prime}\left(\frac{a+b}{2}\right)\right]-K\left(\frac{a+b}{2}\right)\left(\frac{b-a}{2}\right)+\left(f\left(\frac{a+b}{2}\right)-f(a)+K\left(\frac{(a+b)^{2}}{8}-\frac{a^{2}}{2}\right)\right)=\sigma_{1} \\
& \int_{a}^{\frac{a+b}{2}}\left(\frac{a+b}{2}-x\right)\left(K-f^{\prime \prime}(x)\right) d x \\
& =\left(f^{\prime}(a)-K a\right)\left(\frac{b-a}{2}\right)-\left[f\left(\frac{a+b}{2}\right)-f(a)-K\left(\frac{(a+b)^{2}}{8}-\frac{a^{2}}{2}\right)\right]=\sigma_{2} \\
& \int_{a}^{\frac{a+b}{2}}\left(K-f^{\prime \prime}(x)\right) d x=f^{\prime}(a)-f^{\prime}\left(\frac{a+b}{2}\right)+K\left(\frac{b-a}{2}\right)=\sigma_{3} \\
& \int_{\frac{a+b}{2}}^{b}(b-x)\left(K-f^{\prime \prime}(x)\right) d x \\
& =\left[f^{\prime}\left(\frac{a+b}{2}\right)-K\left(\frac{a+b}{2}\right)\right]\left(\frac{b-a}{2}\right)-f(b)+f\left(\frac{a+b}{2}\right)+K\left(\frac{b^{2}}{2}-\frac{(a+b)^{2}}{8}\right)=\sigma_{4} \\
& \int_{\frac{a+b}{2}}^{b}\left(x-\frac{a+b}{2}\right)\left(K-f^{\prime \prime}(x)\right) d x \\
& =\left(K b-f^{\prime}(b)\right)\left(\frac{b-a}{2}\right)+\left[f(b)-f\left(\frac{a+b}{2}\right)-k\left(\frac{(a+b)^{2}}{8}-\frac{b^{2}}{2}\right)\right]=\sigma_{5} \\
& \int_{\frac{a+b}{2}}^{b}\left(K-f^{\prime \prime}(x)\right) d x=f^{\prime}\left(\frac{a+b}{2}\right)-f^{\prime}(b)+K\left(\frac{b-a}{2}\right)=\sigma_{6} .
\end{aligned}
$$


Substituting equalities (12), (13), (14) in inequality (8) and from (4), we have

$$
I_{1} \leq k \frac{(b-a)^{2}}{96}+\frac{1}{2(b-a)}\left(\frac{\alpha_{1} \alpha_{2}}{\alpha_{3}}\right) .
$$

Substituting equalities (15), (16), (17) in inequality (9) and from (6), we have

$$
I_{2} \leq k \frac{(b-a)^{2}}{96}+\frac{1}{2(b-a)}\left(\frac{\alpha_{4} \alpha_{5}}{\alpha_{6}}\right) .
$$

Adding (24) and (25), we obtain

$$
I_{1}+I_{2} \leq k \frac{(b-a)^{2}}{48}+\frac{1}{2(b-a)}\left(\frac{\alpha_{1} \alpha_{2}}{\alpha_{3}}+\frac{\alpha_{4} \alpha_{5}}{\alpha_{6}}\right) .
$$

Substituting equalities (18), (19), (20) in inequality (10) and from (5), we have

$$
I_{1} \geq K \frac{(b-a)^{2}}{96}-\frac{1}{2(b-a)}\left(\frac{\sigma_{1} \sigma_{2}}{\sigma_{3}}\right) .
$$

Substituting equalities (21), (22), (23) in inequality (11) and from (7), we have

$$
I_{2} \geq K \frac{(b-a)^{2}}{96}-\frac{1}{2(b-a)}\left(\frac{\sigma_{4} \sigma_{5}}{\sigma_{6}}\right) .
$$

Adding (27) and (28), we obtain

$$
I_{1}+I_{2} \geq K \frac{(b-a)^{2}}{48}-\frac{1}{2(b-a)}\left(\frac{\sigma_{1} \sigma_{2}}{\sigma_{3}}+\frac{\sigma_{4} \sigma_{5}}{\sigma_{6}}\right) .
$$

From (26) and (29), we have,

$$
K \frac{(b-a)^{2}}{48}-\frac{1}{2(b-a)}\left(\frac{\sigma_{1} \sigma_{2}}{\sigma_{3}}+\frac{\sigma_{4} \sigma_{5}}{\sigma_{6}}\right) \leq I_{1}+I_{2} \leq k \frac{(b-a)^{2}}{48}+\frac{1}{2(b-a)}\left(\frac{\alpha_{1} \alpha_{2}}{\alpha_{3}}+\frac{\alpha_{4} \alpha_{5}}{\alpha_{6}}\right)
$$

where,

$$
I_{1}+I_{2}=\frac{1}{2}\left[f\left(\frac{a+b}{2}\right)+\frac{f(a)+f(b)}{2}\right]-\frac{1}{b-a} \int_{a}^{b} f(x) d x .
$$

And the theorem is proved.

\section{Applications to special means}

We shall consider the means for arbitrary real numbers $\alpha, \beta, \alpha \neq \beta$. We take

$$
\begin{aligned}
H(\alpha, \beta) & =\frac{2}{\frac{1}{\alpha}+\frac{1}{\beta}}, \quad \alpha, \beta \in R \backslash\{0\}, & & \text { (harmonic mean) } \\
A(\alpha, \beta) & =\frac{\alpha+\beta}{2}, \quad \alpha, \beta \in R, & & \text { (arithmetic mean) } \\
L_{n}(\alpha, \beta) & =\left[\frac{\beta^{n+1}-\alpha^{n+1}}{(n+1)(\beta-\alpha)}\right]^{1 / n}, n \in Z \backslash\{-1,0\}, \alpha, \beta \in R, \alpha \neq \beta, & & \text { (generalized log-mean). }
\end{aligned}
$$

Now, using the results of Section 2, we give some applications to special means of real numbers. 
Proposition 1. Let $0<a<b$ and $n \in Z-\{-1,0\}$. Then we have the inequality,

$$
\begin{gathered}
\frac{(b-a)^{2}}{32} n(n+1)\left[A\left(\left(\frac{3 a+b}{4}\right)^{-n-2},\left(\frac{a+3 b}{4}\right)^{-n-2}\right)\right] \leq N \\
\quad \leq \frac{(b-a)^{2}}{64} n(n+1)\left[A\left(\left(\frac{3 a+b}{4}\right)^{-n-2},\left(\frac{a+3 b}{4}\right)^{-n-2}\right)\right]
\end{gathered}
$$

where $N=\frac{1}{2}\left[A^{-n}(a, b)+A\left(a^{-n}, b^{-n}\right)\right]-L_{-n}^{-n}(a, b)$.

Proof. The assertion follows from Theorem 1 applied for $f(x)=x^{-n}, n \in Z-\{-1,0\}$.

Proposition 2. Let $0<a<b$ and $n \in Z-\{-1,0\}$. Then we have the inequality, for all $p>1$ and $\frac{1}{p}+\frac{1}{q}=1$,

$$
\left|L_{-n}^{-n}(a, b)-\frac{1}{2}\left[A^{-n}(a, b)+A\left(a^{-n}, b^{-n}\right)\right]\right| \leq \frac{(b-a)^{2}}{2^{(2 p+1) / p}}|n(n+1)|[B(p+1, p+1)]^{1 / p} L_{-n-q}^{\frac{-n-q}{q}}(a, b)
$$

where $B(p, q)$ is Euler's Beta function.

Proof. If we apply Theorem 2 for $f(x)=x^{-n}$ on $[a, b]$, we obtain that

$$
\begin{aligned}
& \left|\frac{1}{2}\left[A^{-n}(a, b)+A\left(a^{-n}, b^{-n}\right)\right]-L_{-n}^{-n}(a, b)\right| \\
& \quad \leq \frac{(b-a)^{(p+1) / p}}{2^{(2 p+1) / p}}[B(p+1, p+1)]^{1 / p}\left(\int_{a}^{b}\left[-n(-n-1) x^{-n-2}\right]^{q} d x\right)^{1 / q} .
\end{aligned}
$$

Using the facts that

$$
\int_{a}^{b} x^{-n q-2 q} d x=\frac{b^{-n q-2 q+1}-a^{-n q-2 q+1}}{-n q-2 q+1}=\frac{b^{-n-q+1}-a^{-n-q+1}}{-n-q+1}=L_{-n-q}^{-n-q}(a, b)(b-a)
$$

we find that

$$
\begin{aligned}
0 & \leq \frac{1}{2}\left[A^{-n}(a, b)+A\left(a^{-n}, b^{-n}\right)\right]-L_{-n}^{-n}(a, b) \\
& \leq \frac{(b-a)^{(p+1) / p}}{2^{(2 p+1) / p}} n(n+1)[B(p+1, p+1)]^{1 / p} L_{-n-q}^{\frac{-n-q}{q}}(a, b)(b-a)^{1 / q} \\
& \leq \frac{(b-a)^{2}}{2^{(2 p+1) / p}} n(n+1)[B(p+1, p+1)]^{1 / p} L_{-n-q}^{\frac{-n-q}{q}}(a, b) .
\end{aligned}
$$

Hence, we have the conclusion.

Proposition 3. Let $0<a<b$. Then we have the inequality

$$
\left|L_{-2}^{-2}(a, b)-\frac{1}{2}\left[A^{-2}(a, b)+A\left(a^{-2}, b^{-2}\right)\right]-\frac{1}{24}(b-a) H^{-1}\left(a^{3},-b^{3}\right)\right| \leq \frac{3}{32}(b-a)^{2} H^{-1}\left(a^{4},-b^{4}\right) .
$$


Proof. If we apply Theorem 3 for the convex mapping $f(x)=\frac{1}{x^{2}}$ and from the equality $f^{\prime \prime}(x)=$ $\frac{6}{x^{4}}$, we have

$$
\gamma=\frac{6}{\left(\frac{a+b}{2}\right)^{4}} \leq f^{\prime \prime}(x) \leq \frac{6}{a^{4}}=\mu, \quad x \in\left[a, \frac{a+b}{2}\right)
$$

and

$$
\gamma^{\prime}=\frac{6}{b^{4}} \leq f^{\prime \prime}(x) \leq \frac{6}{\left(\frac{a+b}{2}\right)^{4}}=\mu^{\prime}=\gamma, \quad x \in\left[\frac{a+b}{2}, b\right] .
$$

By inequality (3), we obtain,

$$
\left|L_{-2}^{-2}(a, b)-\frac{1}{2}\left[A^{-2}(a, b)+A\left(a^{-2}, b^{-2}\right)\right]-\frac{1}{24}(b-a) H^{-1}\left(a^{3},-b^{3}\right)\right| \leq \frac{3}{32}(b-a)^{2} H^{-1}\left(a^{4},-b^{4}\right) .
$$

Hence, we have the conclusion.

\section{Acknowledgement}

The author wishes to thank one of the referees for his comments.

\section{References}

[1] S. S. Dragomir, Selected Topics on Hermite-Hadamard Inequalities and Applications, http://rgmia.vu.edu.au/SSDragomirWeb.html.

[2] U. S. Kirmaci, M. K. Bakula, M. E. Özdemir and J. E. Pečarić, Hadamard-type inequalities for s-convex functions, Appl. Math. Comput., 193 (2007), 26-35.

[3] U. S. Kirmaci, Improvement and further generalization of inequalities for differentiable mappings and applications, Comput. Math. Appl., 55 (2008), 485-493.

[4] U. S. Kirmaci, Inequalities for differentiable mappings and applications to special means of real numbers and to midpoint formula, Appl. Math. Comput., 147(2004), 137-146.

[5] U. S. Kirmaci and M. E. Özdemir, On some inequalities for differentiable mappings and applications to special means of real numbers and to midpoint formula, Appl. Math. Comput., 153 (2004), 361-368.

[6] U. S. Kirmaci and M. E. Özdemir, Some inequalities for mappings whose derivatives are bounded and applications to special means of real numbers, Appl. Math. Lett., 17 (2004), 641-645.

[7] D.S. Mitrinović Analytic Inequalities, Springer-Verlag New-York, Heidelberg, Berlin, 1970.

K. K. Education Faculty Department of Mathematics, Atatürk University, 25240 Erzurum, Turkey.

E-mail: kirmaci@atauni.edu.tr

K. K. Education Faculty Department of Mathematics, Atatürk University, 25240 Erzurum, Turkey.

E-mail: rdikici@atauni.edu.tr 